\title{
The impact of high-intensity interval exercise training on NK-cell function and circulating myokines for breast cancer prevention among women at high risk for breast cancer
}

\author{
Adriana M. Coletta ${ }^{1,2,12}$ - Nadia H. Agha ${ }^{3} \cdot$ Forrest L. Baker $^{4,5} \cdot$ Grace M. Niemiro $^{4}$ - Preteesh L. Mylabathula ${ }^{4,5}$. \\ Abenaa M. Brewster ${ }^{6} \cdot$ Therese B. Bevers $^{6}$. Enrique Fuentes-Mattei ${ }^{7} \cdot$ Karen Basen-Engquist $^{8} \cdot$ Susan C. Gilchrist ${ }^{6,9}$. \\ Richard J. Simpson $4,5,8,10,11$
}

Received: 26 October 2020 / Accepted: 21 January 2021 / Published online: 8 February 2021

(c) The Author(s) 2021

\begin{abstract}
Purpose Preclinical evidence suggests that natural killer cell (NK-cell) function and myokines facilitate the protective effects of exercise for breast cancer prevention. Since higher-intensity exercise acutely promotes greater mobilization and larger changes in NK-cell cytotoxicity than lower-intensity, high-intensity interval training (HIIT) might offer increased immune protection compared to moderate-intensity continuous-training (MICT). This study compared a 12-week HIIT program to a 12-week MICT program and usual care on changes in resting NK-cell function and circulating myokines among women at high risk for breast cancer.

Methods Thirty-three women were randomized to HIIT, MICT, or usual care, for a supervised exercise intervention. Blood was collected at baseline and end-of-study. The cytotoxic activity of CD3-/CD56+ NK-cells against the K562 target cell line in vitro was determined by flow cytometry. Circulating myokines (IL-15, IL-6, irisin, OSM, osteonectin, IL-7) were assessed with luminex multiplex assays and ELISA. One-way ANOVA and paired sample t-tests assessed between- and within-group differences, respectively. Pearson correlation coefficients determined relationships between baseline fitness and change variables.

Results Significant differences were not observed between groups for change in NK-cell function or circulating myokines $(p>0.05)$. Significant correlations were only observed for baseline peak aerobic capacity $(\mathrm{ml} / \mathrm{kg} / \mathrm{min})$ and change in NKcell-specific lysis $(r=-0.43, p=0.02)$ and hemacytotoxicity for the total sample $(r=-0.46, p=0.01)$.

Conclusion Our findings suggest that exercise intensity may not significantly impact change in resting NK-cell function and circulating myokines among women at high risk for breast cancer. Structured exercise training may have a larger impact on NK-cell function in those with lower levels of cardiorespiratory fitness.
\end{abstract}

Clinical trial registration: NCT02923401; Registered on October 4, 2016

Keywords Exercise immunology $\cdot$ Cytokines $\cdot$ Breast cancer prevention

\section{Introduction}

High-intensity interval exercise training (HIIT), compared to traditional moderate-intensity continuous aerobic exercise training (MICT), promotes favorable changes in markers of health, notably cardiorespiratory fitness (CRF), cardiac function, blood lipids, oxidative stress, inflammation, insulin

Adriana M. Coletta

adriana.coletta@hci.utah.edu

Extended author information available on the last page of the article sensitivity, and quality of life, among apparently healthy and clinical populations [1-4]. HIIT requires less time to achieve the same exercise dose (i.e., metabolic equivalent hours) compared to MICT, which is advantageous because time is the biggest reported barrier to engaging in regular exercise among both apparently healthy and clinical populations [5]. In addition to the health benefits and time efficiency of HIIT, high-intensity exercise exhibits utility in the context of breast cancer prevention, such that greater than $6 \mathrm{~h}$ per week of vigorous-intensity physical activity over a lifetime is associated with a $23 \%$ reduction in breast cancer risk [6]. Breast cancer is the most common cancer among women 
worldwide, and the majority of cases occur in postmenopausal women [7, 8]. In addition to menopausal status, obesity, a history of non-invasive breast cancer (i.e., ductal carcinoma in situ or lobular carcinoma in situ), and an elevated Gail Model risk score, significantly increase a woman's risk of developing breast cancer $[9,10]$.

There are multiple proposed mechanisms that underpin the association between exercise and breast cancer risk. Of particular interest to HIT is the interplay of mobilization, redistribution, and effector functions of lymphocytes, particularly natural killer cells (NK-cells), and muscle-derived cytokines (e.g., 'myokines') released from skeletal muscle during contraction [11-13]. A recent review by Duggal et al. [14] suggests an association between exercise-induced myokine release and subsequent changes in the composition and function of the peripheral leukocyte compartment. Specific to breast cancer, in vitro evidence demonstrates that the myokine irisin suppresses malignant breast epithelial cell number and migration [15], and oncostatin M (OSM) reduces cellular proliferation and increases apoptosis [11]. Evidence from human studies exhibit an acute increase in irisin following exercise, and CRF is a strong predictor of the magnitude of change of irisin expression after acute exercise [16]. Observational evidence suggests a protective effect between elevated irisin levels at rest and risk of invasive breast cancer [17].

In coordination with immune function, IL-15 facilitates the expansion and maintenance of NK-cells and effector T-cells [18], while IL-6 has been shown to promote trafficking and tumor infiltration of exercise-mobilized NK-cells in several murine cancer models [19]. The cytotoxic activity of NK-cells against various cancer cell lines has been found to increase in response to both acute and chronic exercise, which has been attributed to phenotypic shifts in NK-cells and their frequent mobilization and redistribution with each bout of exercise [20, 21]. Because higher-intensity exercise acutely promotes greater mobilization and larger change in NK-cell cytotoxicity than lower-intensity exercise [20], HIIT might offer increased levels of immune protection in postmenopausal women with obesity at high risk of breast cancer compared to MICT. Moreover, a recent case-cohort study observed an inverse association between breast cancer risk and higher resting levels of NK-cells among postmenopausal women, although the association was not statistically significant [22].

We previously compared 12 weeks of HIIT, to 12 weeks of MICT, and usual care (non-exercise control) among postmenopausal women with obesity considered at high risk of breast cancer [2]. We observed significantly greater improvements in CRF variables with HIIT compared to MICT and usual care (UC) [2]. The purpose of this study was to determine the impact of this 12-week HIIT program compared with MICT and usual care on changes in resting NK-cell function and circulating myokines among women at high risk for breast cancer.

\section{Materials and methods}

\section{Study design, participants, recruitment, and intervention procedures}

This was a randomized controlled trial, where randomization was stratified by body mass index (BMI). Participants were randomized to HIIT, MICT, or UC for a 12-week intervention. This investigation was approved by the University of Texas MD Anderson Cancer Center Institutional Review Board. Details regarding eligibility criteria, recruitment, and intervention procedures are reported elsewhere [2].

Briefly, eligible participants consisted of postmenopausal women with overweight or obesity status, as defined by body mass index $\left(\geq 25 \mathrm{~kg} / \mathrm{m}^{2}\right)$, who were considered at heightened risk of developing breast cancer due to an elevated Gail 5-year risk score (>1.66\%), lifetime risk score ( $>20 \%)$, history of ductal or lobular atypia, or history of ductal or lobular carcinoma in situ (non-invasive breast cancer). Participants were recruited from the University of Texas MD Anderson Cancer Center, Clinical Cancer Prevention Center.

Participants assigned to HIIT and MICT presented at the Cancer Prevention Center three times per week and completed their supervised exercise sessions on a treadmill. HIIT consisted of an aerobic interval training protocol. Following a 5-min warm-up at 50-70\% peak heart rate (HR), four 4-min high-intensity intervals were completed at 90-100\% peak HR, with each high-intensity interval followed by a 3 -min active recovery interval at $50-70 \%$ peak HR. The HIIT workout was $33 \mathrm{~min}$ in length. The MICT workout was $41 \mathrm{~min}$ in length, completed at $60-70 \%$ peak HR. Participants assigned to UC received educational material related to healthful diet and exercise habits at baseline, and monthly phone calls by study personnel pertaining to their healthy lifestyle goals.

\section{Assessment procedures}

Details regarding assessment procedures are reported elsewhere [2]. Briefly, assessment sessions were conducted at baseline, 6 weeks and upon completion of the 12-week intervention (end-of-study). Relevant to the present investigation, cardiopulmonary exercise testing was conducted at baseline, 6 weeks and end-of-study, utilizing the modified Balke protocol [23], to measure CRF variables. Peak HR measured from the cardiopulmonary exercise testing was used to prescribe individualized HR training zones. Additionally, fasting whole blood was collected at baseline and end-of-study using standard phlebotomy procedures. The 
median and average number of days between last workout and end-of-study assessment blood draw were 3 and 5 days, respectively. Upon completion of the last training session and prior to the participant's end-of-study assessment, participants were instructed to continue their normal diet and lifestyle.

\section{NK-cell function analysis}

At baseline and end-of-study, fasting whole blood was collected into one $8.5 \mathrm{~mL}$ ACD-containing collection tube, one $6 \mathrm{~mL}$ lithium heparin-containing collection tube, and one $2.5 \mathrm{~mL}$ EDTA-containing collecting tube. Peripheral blood mononuclear cells (PBMCs) were isolated from $8.5 \mathrm{~mL}$ whole blood by density gradient centrifugation (Histopaque-1077, Sigma-Aldrich). The HLA-deficient leukemia cell line K562 (ATCC: CCL-143) was maintained in a glutamine-enriched, $10 \%$ FBS and $1 \%$ penicillin streptomycin RPMI-1640 (Sigma-Aldrich) and used as target cells for the cytotoxicity assay. On the day of the NK-cell assay, K562 cells were stained with anti-CD71 FITC antibody (IgG1, Clone OKT9), washed, and re-suspended in media (10\% FBS and 1\% penicillin streptomycin RPMI-1640). Monocyte-depleted PBMCs were co-cultured with CD71-labeled K562 cells at 0:1 (spontaneous cell death), 1:1, 5:1, 10:1, and 20:1 effector (PBMCs): target (K562) cell ratios for $4 \mathrm{~h}$ in a humidified $\mathrm{CO} 2$ incubator at $37{ }^{\circ} \mathrm{C}$. The same number of PBMCS and target cells were used for baseline and end-of-study samples. NK-cells were quantified as a percentage of isolated PBMCs [24]. NK-cell cytotoxicity was determined by flow cytometry as previously described [25]. Hemacytotoxicity was defined as the specific lysis (\% total lysis - \% spontaneous death) of 10,000 target cells from 50 $\mathrm{uL}$ of whole blood. Overall, the following variables were used to assess NK-cell function: NK-cells as percent of lymphocytes, NK-cell counts (cell/uL), hemacytotoxicity, lytic index, and specific lysis.

\section{Myokine analysis}

\section{Irisin, Oncostatin M (OSM), IL-15, IL-6, Osteonectin}

Fasting whole blood was collected into one, 6 mL EDTAcontaining collection tube at baseline and end-of-study. Once blood was collected, the EDTA tube was centrifuged at $1000 \mathrm{~g}$ for $10 \mathrm{~min}$ and plasma was then aliquoted in $1.5 \mathrm{~mL}$ microcentrifuge tubes and stored at $-80{ }^{\circ} \mathrm{C}$ until completion of the trial for batch analysis. Upon completion of the trial, plasma samples were analyzed via a customized, Luminex ${ }^{\circledR}$ Human Myokine Magnetic Bead Panel (HMYOMAG-56 K, Millipore Corporation, Billerica MA) using a Luminex $200^{\mathrm{TM}}$ System with Luminex YX Platform ${ }^{\mathrm{TM}}$ software. In brief, $35 \mu \mathrm{L}$ of plasma were diluted 1:2 with $35 \mu \mathrm{L}$ of Assay
Buffer and $25 \mu \mathrm{L}$ of diluted sample were run in duplicates following the kit-optimized protocol recommended by the company (Millipore Corporation, Billerica MA). The following myokines were included in the panel: irisin, OSM, IL-15, IL-6, and osteonectin. The intra-assay coefficient of variation for these panels are $<10 \%$. Some myokines within the luminex multiple assay resulted in undetectable levels. Undetectable levels were defined as follows: IL- $15<2.44 \mathrm{pg} / \mathrm{mL}$, IL- $6<0.59 \mathrm{pg} / \mathrm{mL}$, irisin $<244.14 \mathrm{pg} /$ $\mathrm{mL}$, and osteonectin $<7.32 \mathrm{pg} / \mathrm{mL}$. For IL-15, three samples included undetectable levels in the HIIT, MICT, and usual care. For IL-6 only one sample included undetectable levels for MICT. For irisin, one sample in MICT and five in usual care contained undetectable levels. Finally, for osteonectin, only one sample in the usual care group contained undetectable levels.

\section{IL-7}

Plasma samples were analyzed in duplicate for IL-7 concentrations via ELISA (R\&D Systems, Minneapolis, MN) following the manufacturer's instructions. The lower level of detection of the kit used was less than $0.1 \mathrm{pg} / \mathrm{mL}$. Samples were read using a SpectraMax M3 plate reader (Molecular Devices, LLC, San Jose CA) and analyzed on SoftMax Pro (v. 6.3, Molecular Devices, LLC, San Jose CA). The intraassay coefficient of variation for this plate was $10.82 \%$. All samples had detectable levels of IL-7 in plasma.

\section{Statistical analysis}

We analyzed our data based on the intention-to-treat principle. One-way analysis of variance (ANOVA) was used to assess differences between groups at baseline and end-ofstudy, and for changes from baseline (end-of-study value - baseline value) for NK-cell function and myokine variables. General linear model univariate analysis of covariance was used to assess within- and between-group differences in changes in NK-cell function and myokine variables, controlling for baseline age and BMI. Paired sample t-tests were used to assess within-group differences from baseline to endof-study. Pearson correlation coefficients were assessed to determine the relationship between: (1) baseline peak aerobic capacity $(\mathrm{ml} / \mathrm{kg} / \mathrm{min})$ and change in both NK-cell function and myokines for the total sample and by group, (2) change in peak aerobic capacity $(\mathrm{ml} / \mathrm{kg} / \mathrm{min})$ and change in NK-cell function and myokines, (3) change in NK-cell function and change in myokines. In addition, Fisher's $r$ to $\mathrm{z}$ transformation was completed, using a two-tailed test of significance, to test for potential differences in correlations between groups for the comparison of baseline peak aerobic capacity and change in NK-cell function and myokines. 
Data were analyzed with IBM® SPSS $®$ Version 26 software (IBM Corp., Armonk, NY, USA).

\section{Results}

\section{Participant characteristics}

At baseline, there were no significant differences between groups among the following variables: age, body mass index, cardiorespiratory fitness, race, ethnicity, status of breast cancer risk (i.e., history of ductal or lobular carcinoma in situ, atypia, elevated risk scores) [2]. Thirty-three participants completed the trial. Twelve participants who completed the intervention were randomized to HIIT, 10 completers to MICT, and 11 completers to UC. Table 1 presents baseline characteristics of the 33 participants who completed the intervention.

\section{NK-cell function}

Table 2 presents group means and statistics for betweengroup differences for NK-cell function variables at baseline, end-of-study, and change from baseline in the unadjusted model. Specific lysis from the 20:1 E:T ratio is reported. Overall, significant differences were not observed within or between groups for NK-cell function variables in unadjusted and adjusted models.

A significant correlation was observed for baseline peak aerobic capacity $(\mathrm{ml} / \mathrm{kg} / \mathrm{min})$ and change in a measure of NK-cell cytotoxicity, specific lysis, in all participants, but not by group (HIIT: $r=-0.39, \mathrm{z}=0.41, p=0.21$; MICT: $r=-0.27, z=0.28, p=0.49$; UC: $r=-0.59, z=0.68$,

Table 1 Baseline characteristics of completers

\begin{tabular}{llll}
\hline & HIIT & MICT & UC \\
& Mean (SD) & Mean (SD) & $62.6(7.0)$ \\
\hline Age (years) & $64.4(6.2)$ & $66.1(13.6)$ & $29.9(2.5)$ \\
BMI $\left(\mathrm{Kg} / \mathrm{m}^{2}\right)$ & $32.3(8.6)$ & $32.0(5.6)$ & $19.4(3.8)$ \\
VO2 peak (ml/kg/min) & $18.8(3.2)$ & $20.8(3.5)$ & $N(\%)$ \\
\hline & $N(\%)$ & $N(\%)$ & $8(73 \%)$ \\
Elevated Gail & $10(84 \%)$ & $9(90 \%)$ & $2(18 \%)$ \\
DCIS & $1(8 \%)$ & 0 & 0 \\
LCIS & $1(8 \%)$ & 0 & $1(9 \%)$ \\
Atypia & 0 & $1(10 \%)$ & $4.31(4.31)$ \\
Gail Risk Score $(n=27)$ & $3.25(1.90)$ & $2.68(0.74)$ & \\
\hline
\end{tabular}

Table 2 Differences between groups in NK-cell function

\begin{tabular}{|c|c|c|c|c|c|c|c|c|}
\hline & & $N$ & Baseline & $p$-level & End-of-Study & $p$-level & Change from Baseline & $p$-level \\
\hline \multirow[t]{3}{*}{ NK-Cells (\%Lymphocytes) } & HIIT & 12 & $9.56 \pm 5.04$ & \multirow[t]{3}{*}{0.70} & $10.50 \pm 3.60$ & \multirow[t]{3}{*}{0.66} & $0.94 \pm 4.99$ & \multirow[t]{3}{*}{0.98} \\
\hline & MICT & 10 & $8.24 \pm 5.27$ & & $8.80 \pm 7.31$ & & $0.56 \pm 4.70$ & \\
\hline & UC & 11 & $7.95 \pm 4.17$ & & $8.66 \pm 4.86$ & & $0.71 \pm 3.57$ & \\
\hline \multirow[t]{3}{*}{ NK-Cell Counts (cell/uL) } & HIIT & 12 & $175.18 \pm 88.68$ & \multirow[t]{3}{*}{0.48} & $188.15 \pm 54.44$ & \multirow[t]{3}{*}{0.50} & $12.98 \pm 87.97$ & \multirow[t]{3}{*}{0.99} \\
\hline & MICT & 10 & $134.44 \pm 75.23$ & & $146.30 \pm 94.99$ & & $11.86 \pm 79.28$ & \\
\hline & UC & 11 & $145.12 \pm 78.90$ & & $161.52 \pm 99.09$ & & $16.40 \pm 54.04$ & \\
\hline \multirow[t]{3}{*}{ Hemacytotoxicity (\#dead cells/50 uL blood) } & HIIT & 12 & $20.60 \pm 10.20$ & \multirow[t]{3}{*}{0.70} & $20.08 \pm 12.96$ & \multirow[t]{3}{*}{0.80} & $-0.52 \pm 10.04$ & \multirow[t]{3}{*}{0.89} \\
\hline & MICT & 10 & $17.84 \pm 10.68$ & & $16.87 \pm 9.81$ & & $-0.97 \pm 5.28$ & \\
\hline & UC & 11 & $17.45 \pm 8.38$ & & $18.11 \pm 11.18$ & & $0.65 \pm 7.95$ & \\
\hline \multirow[t]{3}{*}{ Lytic Index (\#dead target cells/NK-cell) } & HIIT & 12 & $412.18 \pm 239.62$ & \multirow{3}{*}{0.89} & $344.40 \pm 226.41$ & \multirow{3}{*}{0.90} & $-67.78 \pm 237.86$ & \multirow[t]{3}{*}{0.43} \\
\hline & MICT & 10 & $350.34 \pm 270.56$ & & $393.46 \pm 377.58$ & & $43.12 \pm 205.38$ & \\
\hline & $\mathrm{UC}$ & 11 & $403.25 \pm 403.06$ & & $341.32 \pm 268.03$ & & $-61.93 \pm 205.40$ & \\
\hline \multirow{3}{*}{$\begin{array}{l}\text { Specific Lysis (\%killed target cells/10,000 } \\
\text { NK-cells) }\end{array}$} & HIIT & 12 & $23.71 \pm 13.19$ & \multirow[t]{3}{*}{0.75} & $27.58 \pm 15.41$ & \multirow[t]{3}{*}{0.84} & $3.87 \pm 13.11$ & \multirow[t]{3}{*}{0.24} \\
\hline & MICT & 10 & $28.41 \pm 20.90$ & & $24.76 \pm 13.72$ & & $-3.65 \pm 11.78$ & \\
\hline & $\mathrm{UC}$ & 11 & $24.12 \pm 12.27$ & & $28.63 \pm 17.07$ & & $4.51 \pm 10.90$ & \\
\hline
\end{tabular}


$p=0.07)$. A significant correlation was also observed for baseline peak aerobic capacity $(\mathrm{ml} / \mathrm{kg} / \mathrm{min})$ and a marker of NK-cell function, hemacytotoxicity, for the total sample $(r=-0.46, p=0.01, n=31)$ and in UC $(r=-0.70$, $z=0.87, p=0.02)$, but not in HIIT $(r=-0.54, z=0.60$, $p=0.07)$ or MICT $(r=-0.02, z=0.02, p=0.96)$. Significant correlations were not observed for the total sample or by group for NK-cells as percent lymphocyte $(r=0.05$, $p=0.81$; HIIT: $r=0.49, z=0.54, p=0.11$; MICT: $r=-0.40$, $z=0.42, p=0.29$; UC: $r=-0.05, z=0.05, p=0.90)$, NK-cell counts $(r=0.04, p=0.82$; HIIT: $r=0.54, z=0.60, p=0.07$; MICT: $r=-0.47, z=0.51, p=0.20$; UC: $r=-0.12$, $z=0.12, p=0.73)$, or lytic index $(r=-0.31, p=0.10$; HIIT: $r=-0.57, z=0.65, p=0.05$; MICT: $r=0.35, z=0.37$, $p=0.36$; UC: $r=-0.43, z=0.46, p=0.21$ ) (Fig. 1).

Based on the z-score differences for each group, significant differences in correlations of baseline peak aerobic capacity and change in markers of NK-cell function between groups were not significant $(p>0.05$; specific lysis: HIIT-vs-MICT $=0.27$, MICT-vs-UC $=-0.77$, HIITvs-UC $=-0.55$; hemacytotoxicity: HIIT-vs-MICT $=1.16$, MICT-vs-UC $=-1.64$, HIIT-vs-UC $=-0.54$; percent lymphocyte: HIIT-vs-MICT $=0.22$, MICT-vs-UC $=0.72$, HIIT-vs-UC $=1.00$; NK-cell count: HIIT-vs-MICT $=0.19$, MICT-vs-UC $=0.75$, HIIT-vs-UC $=1.00 ;$ lytic index: HIIT-vs-MICT $=0.56$, MICT-vs-UC $=-0.18$, HIIT-vs$\mathrm{UC}=0.39)$. Significant correlations were not observed between change in peak aerobic capacity and change in NK-cell function.

Correlation analyses between change in markers of NKcell function and change in myokines by total sample reveal a significant relationship between change in irisin and specific lysis $(r=0.42, p=0.04, n=25)$ and change in IL-6 and lytic index ( $r=0.41, p=0.02, n=25)$. By group, HIIT $(n=12)$ revealed significant correlations between change in IL-7 and change in both percent lymphocytes $(r=0.72$, $p=0.009)$, and NK-cell counts $(r=-0.66, p=0.02)$, change in IL-6 and change in hemacytotoxicity $(r=0.61, p=0.03)$, lytic index $(r=0.66, p=0.02)$, and specific lysis $(r=0.81$, $p=0.001)$. MICT only revealed significant correlations between change in IL-7 and change in percent lymphocytes $(r=0.91, p<0.001)$ and NK-cell counts $(r=0.83, p=0.003)$. UC revealed significant correlations between change in IL-7 and change in both NK-cell counts $(r=0.62, p=0.04)$ and lytic index $(r=0.68, p=0.02)$. No other significant correlations were observed.

\section{Myokines}

Table 3 presents group means and statistics for betweengroup differences for myokines at baseline, end-of-study, and change from baseline in the unadjusted model. Significant differences between groups were not observed at baseline and end-of-study ( $p>0.05)$. Change from baseline was not significantly different between groups in the unadjusted and adjusted model. Within-group differences were only observed in usual care for IL-6 in the unadjusted model, such that participants exhibited a significant reduction in IL-6 from baseline to end-of-study $(-1.34 \pm 1.74, p=0.03)$. No other significant within-group differences were observed for myokines in the unadjusted and adjusted model.

Regarding correlation analyses, baseline peak aerobic capacity $(\mathrm{ml} / \mathrm{kg} / \mathrm{min})$ was not associated with change in IL-15 ( $r=-0.13, p=0.55, n=23)$, IL-6 $(r=-0.28, p=0.14$, $n=30)$, IL-7 $(r=-0.26, p=0.15, n=31)$, irisin $(r=-0.34$, $p=0.11, n=23)$, OSM $(r=-0.13, p=0.48, n=31)$, or osteonectin $(r=-0.04, p=0.85, n=31)$ for the total sample. Significant associations were also not observed by group for IL-15 (HIIT: $r=-0.27, z=0.26, p=0.49$; MICT: $r=0.37$, $z=0.35, p=0.47$; UC: $r=-0.28, z=0.28, p=0.51$ ), IL-6 (HIIT: $r=-0.50, z=0.55, p=0.10$; MICT: $r=0.05$, $z=0.05, p=0.91$; UC: $r=-0.25, z=0.26, p=0.49)$, IL-7 (HIIT: $r=-0.47, z=0.51, p=0.17$; MICT: $r=-0.26$, $z=0.26, p=0.49$; UC: $r=-0.50, z=0.55, p=0.14)$, iri$\sin$ (HIT: $r=-0.28, z=0.29, p=0.44$; MICT: $r=-0.37$, $z=0.39, p=0.37$; UC: $r=-0.39, z=0.41, p=0.52)$, OSM (HIIT: $-0.38, z=0.40, p=0.22$; MICT: $r=0.43, z=0.46$, $p=0.24$; UC: $r=-0.23, z=0.23, p=0.53$ ), or osteonectin (HIIT: $0.21, z=0.21, p=0.51$; MICT: $r=0.26, z=0.27$, $p=0.50$; UC: $r=-0.50, z=0.55, p=0.15$ ).

Based on the z-score differences for each group, significant differences in correlations of baseline peak aerobic capacity and change in myokines between groups were not significant $(p>0.05$; IL-15: HIIT-vs-MICT $=-0.14$, MICT-vs-UC $=0.10$, HIIT-vs-UC $=-0.03$; IL-6: HIIT-vsMICT $=0.95$, MICT-vs-UC $=-0.38$, HIIT-vs-UC $=0.60$; IL-7: HIIT-vs-MICT $=0.48$, MICT-vs-UC $=-0.53$, HIITvs-UC $=-0.08$; irisin: HIIT-vs-MICT $=-0.18$, MICTvs-UC $=-0.03$, HIIT-vs-UC: $-0.18 ;$ OSM: HIIT-vsMICT $=-0.12$, MICT-vs-UC $=0.44$, HIIT-vs-UC $=0.34$; osteonectin: HIIT-vs-MICT $=-0.10$, MICT-vs-UC $=-0.53$, HIIT-vs-UC $=-0.67$ ). Significant correlations were not observed between change in peak aerobic capacity and change in myokines.

\section{Discussion}

We aimed to compare the impact of a 12-week high-intensity interval training (HIIT) program to a 12 -week moderateintensity continuous aerobic training (MICT) program on changes in resting NK-cell function and circulating myokines among postmenopausal women with obesity at heightened risk for breast cancer. We previously reported that this trial demonstrated low attrition rates, and high adherence and compliance rates (adherence: $90 \%$ in HIIT 

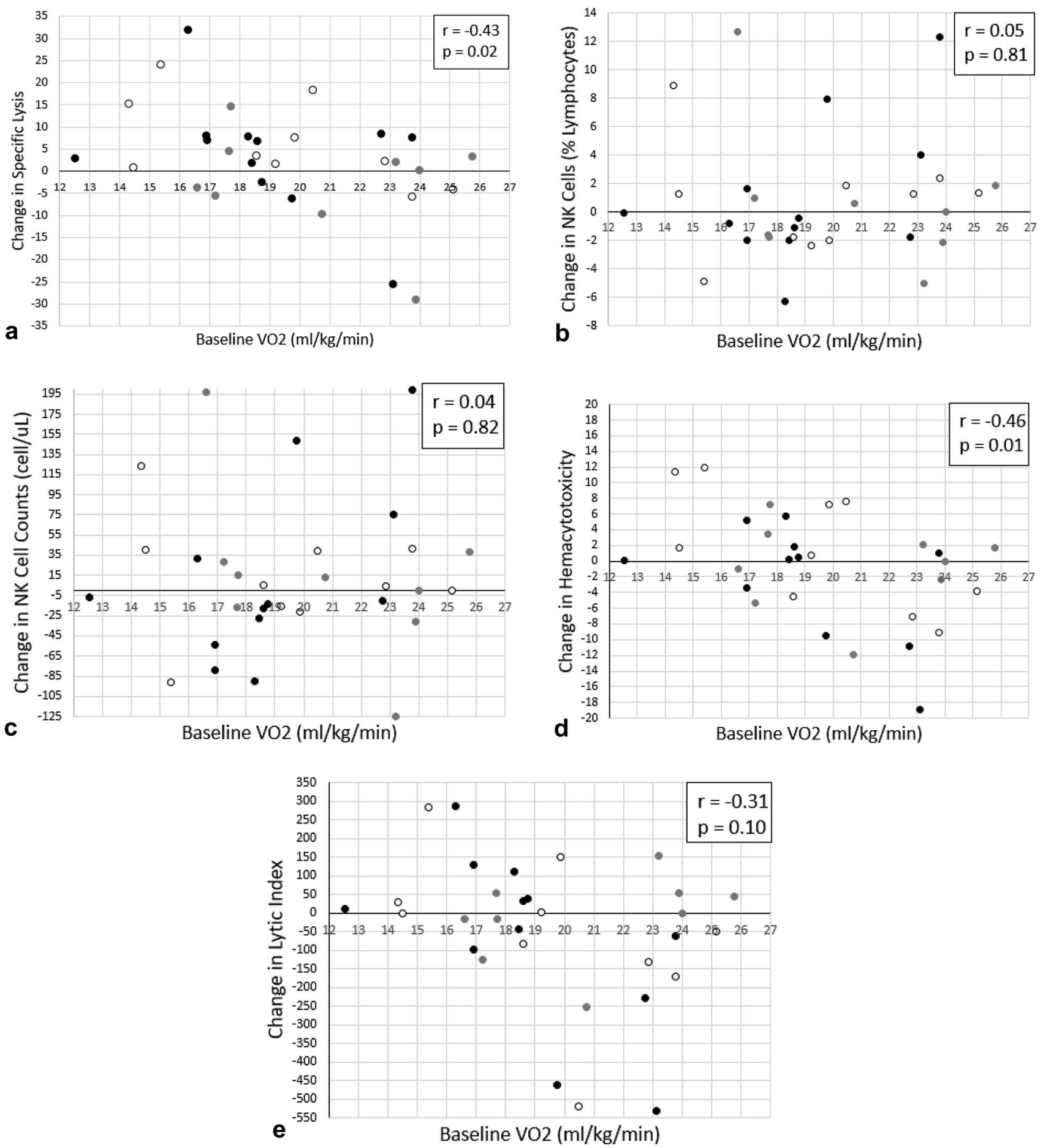

Fig. 1 Correlation between baseline peak aerobic capacity $(\mathrm{ml} / \mathrm{kg} / \mathrm{min})$ and change in markers of NK-Cell function for the total sample. a Specific lysis, b percent lymphocytes, c NK-cell counts, d hemacytotoxicity, e Lytic Index. HIIT black circles, MICT gray circles, UC white circles

and $89 \%$ in MICT; compliance: $100 \%$ to HIIT and MICT protocols), and greater improvements in cardiorespiratory fitness (CRF) after 12 weeks of HIIT compared to MICT and usual care [2], yet in the present investigation, we did not observe significant changes in resting NK-cell function following 12 weeks of training. This suggests that exercise training, regardless of intensity, may not influence the ability of NK-cells to kill tumor target cells in vitro. While we observed an increase in NK-cell counts across all groups, these findings are consistent with a recent 12 -week exercise 
Table 3 Differences between groups in myokines

\begin{tabular}{|c|c|c|c|c|c|c|c|c|}
\hline & & $N$ & Baseline & $p$-level & End-of-Study & $p$-level & Change from Baseline & $p$-level \\
\hline \multirow[t]{3}{*}{ IL-15 (pg/mL) } & HIIT & 9 & $5.51 \pm 6.06$ & \multirow[t]{3}{*}{0.14} & $5.01 \pm 5.10$ & \multirow[t]{3}{*}{0.20} & $-0.51 \pm 2.78$ & \multirow[t]{3}{*}{0.17} \\
\hline & MICT & 7 & $5.87 \pm 4.74$ & & $6.53 \pm 5.44$ & & $0.66 \pm 0.97$ & \\
\hline & UC & 8 & $13.89 \pm 13.77$ & & $12.02 \pm 11.33$ & & $-1.87 \pm 3.02$ & \\
\hline \multirow[t]{3}{*}{ IL-6 (pg/mL) } & HIIT & 12 & $7.07 \pm 5.61$ & \multirow[t]{3}{*}{0.68} & $6.14 \pm 5.29$ & \multirow[t]{3}{*}{0.72} & $-0.93 \pm 4.16$ & \multirow[t]{3}{*}{0.91} \\
\hline & MICT & 9 & $5.16 \pm 4.60$ & & $4.37 \pm 3.53$ & & $-0.79 \pm 2.10$ & \\
\hline & UC & 11 & $7.51 \pm 7.73$ & & $6.17 \pm 7.01$ & & $-1.34 \pm 1.74$ & \\
\hline \multirow[t]{3}{*}{ Irisin $(\mathrm{pg} / \mathrm{mL})$} & HIIT & 10 & $323.35 \pm 344.39$ & \multirow[t]{3}{*}{0.21} & $356.69 \pm 339.35$ & \multirow[t]{3}{*}{0.25} & $33.34 \pm 299.39$ & \multirow[t]{3}{*}{0.60} \\
\hline & MICT & 9 & $464.68 \pm 469.68$ & & $375.63 \pm 353.65$ & & $-89.04 \pm 293.10$ & \\
\hline & UC & 6 & $795.59 \pm 735.80$ & & $722.03 \pm 667.03$ & & $-73.56 \pm 202.08$ & \\
\hline \multirow[t]{3}{*}{ OSM (pg/mL) } & HIIT & 12 & $3.87 \pm 1.90$ & \multirow[t]{3}{*}{0.55} & $3.70 \pm 1.89$ & \multirow[t]{3}{*}{0.35} & $-0.17 \pm 1.45$ & \multirow[t]{3}{*}{0.90} \\
\hline & MICT & 10 & $5.18 \pm 5.05$ & & $5.37 \pm 5.59$ & & $0.19 \pm 1.06$ & \\
\hline & $\mathrm{UC}$ & 11 & $6.15 \pm 6.77$ & & $6.27 \pm 4.63$ & & $0.12 \pm 2.85$ & \\
\hline \multirow[t]{3}{*}{ Osteonectin $(\mathrm{pg} / \mathrm{mL})$} & HIIT & 12 & $253.10 \pm 125.95$ & \multirow[t]{3}{*}{0.44} & $264.94 \pm 171.58$ & \multirow[t]{3}{*}{0.90} & $11.85 \pm 77.07$ & \multirow[t]{3}{*}{0.30} \\
\hline & MICT & 10 & $294.52 \pm 107.93$ & & $269.26 \pm 99.30$ & & $-25.25 \pm 45.83$ & \\
\hline & UC & 10 & $235.45 \pm 65.97$ & & $245.69 \pm 59.44$ & & $10.24 \pm 46.60$ & \\
\hline \multirow[t]{3}{*}{ IL-7 (pg/mL) } & HIIT & 12 & $2.57 \pm 4.33$ & \multirow[t]{3}{*}{0.37} & $1.00 \pm 0.71$ & \multirow[t]{3}{*}{0.15} & $-1.57 \pm 4.21$ & \multirow[t]{3}{*}{0.10} \\
\hline & MICT & 10 & $1.44 \pm 1.08$ & & $2.39 \pm 2.52$ & & $0.95 \pm 2.00$ & \\
\hline & $\mathrm{UC}$ & 11 & $0.98 \pm 0.71$ & & $1.47 \pm 1.33$ & & $0.49 \pm 1.37$ & \\
\hline
\end{tabular}

intervention conducted among postmenopausal women with obesity (but not at heightened risk for breast cancer) that compared moderate-intensity continuous aerobic exercise to resistance training and a non-exercise control [26].

Additionally, our findings are similar to a 12-month moderate-intensity continuous aerobic exercise intervention conducted among postmenopausal women with obesity, such that no effects on change in natural killer cell cytotoxicity were observed [27]. Moreover, a recent pilot study in breast cancer patients found no effect of a 12-week resistance exercise training intervention on the gene expression profile of peripheral blood NK-cells [28]. Taken together, these findings suggest that chronic exercise, regardless of intensity, may not impact changes in resting (e.g., $>24 \mathrm{~h}$ since the last exercise bout) NK-cell function in vitro or NKcell gene expression. However, because exercise training has been shown to increase NK-cell trafficking and infiltration of tumors in vivo [19], it is possible that other aspects of NK-cell function are improved with exercise training such as mobilization, tissue migration, tumor infiltration, and the ability to secrete and be activated by various cytokines [18].

Since fitness (e.g., higher CRF) is associated with greater levels of NK-cell function [29-31] and a greater release of myokines into the circulation immediately following exercise [16], we explored the relationship between baseline fitness levels and changes in immune function and myokines following exercise training. We did not observe significant correlations between change in circulating myokines and baseline fitness for the total sample or by group, which suggests that baseline CRF may not impact the magnitude of change of resting levels of myokines after participating in a 12-week exercise program. We did, however, find a significant relationship between baseline CRF levels and the change in NK-cell function, indicating that a positive change in NK-cell function after an exercise training intervention might be more apparent in those with lower CRF at baseline. However, these findings should be interpreted with caution, due to our smaller sample size and exploratory nature of this analysis.

Regarding myokines, we assessed irisin, OSM, osteonectin, IL-6, IL-15, and IL-7 on account of evidence supporting the utility of these myokines in the context of cancer prevention and control [11, 15, 19, 32-34]. Additionally, IL-15 and IL-6 have known roles in activating NK-cell and T-cells during exercise [18] and ability to facilitate the immune system and promote anti-cancer effects in preclinical models [19, 33]. We did not observe significant differences between groups for changes in circulating myokines at rest after participation in a 12-week exercise training program. Among OSM, IL-15, IL-6, and IL-7 we observed either a small reduction in levels from baseline or negligible increase within groups. Together, these findings suggest that exercise intensity may not influence the extent of change in these circulating myokines at rest.

While we did not observe statistically significant differences between or within groups for changes in plasma osteonectin or irisin, we observed a larger extent of change in both directions, such that HIIT was associated with an increase in change in resting levels, and MICT with a decrease. Our findings related to osteonectin are consistent 
with a previous investigation that observed an increase in resting mRNA expression of osteonectin in skeletal muscle after participation in a HIIT program [35]. For irisin, an increase after HIIT aligns with preclinical and human studies demonstrating an increase in gene expression of irisin (also referred to as fibronectin type III domaincontaining protein 5 [FNDC5]) following high-intensity exercise specifically [36, 37]. Evidence in humans has also demonstrated an increase in irisin gene expression at rest for up to 20 days after high-intensity exercise [36]. We also observed a reduction in resting levels of irisin after MICT, which is consistent with a recent meta-analysis conducted by Qui et al. [38].

Interestingly, when comparing the correlations of change in NK-cell function and myokines, participation in HIIT was associated with the most number of correlations in changes in NK-cell function and myokines. These findings suggest the synergistic effects of high-intensity exercise on NK-cell function and myokines. These findings may serve as early evidence related to the underlying physiological mechanisms that associate vigorous-intensity exercise with reductions in breast cancer risk [6].

This is the first study to our knowledge to compare the chronic effects of different types of exercise intensities on changes in resting NK-cell function and circulating myokines among women at heightened risk of breast cancer, who are also postmenopausal with obesity. We consider this a strength of our trial. Other strengths include the supervised exercise intervention and measurement methods used to assess circulating myokines (luminex multiplex panel) and NK-cell function. However, our study is not without limitations. Specific to the myokine analysis, we consider the inconsistent timing for blood collection in relation to last workout across participants a limitation of our study. Additionally, the smaller sample size, use of K562 cell line and only one cell line for NK-cell analyses are also limitations to our findings.

Future studies assessing exercise-induced changes in resting NK-cell function in the context of breast cancer should utilize a broad range of breast cancer cell lines. Additionally, future work should consider assessment of other aspects of NK-cell function in vivo (e.g., mobilization and redistribution), and assessment of baseline CRF as it compares to changes in markers of immune function due to exercise. Future research assessing myokines specifically should consider consistent timing of blood collection in relation to completion of the exercise training program. Furthermore, findings from this exploratory analysis provide preliminary estimates that can be used in future studies for power calculation. In summary, our findings suggest that exercise intensity does not significantly impact resting in vitro NK-cell function and change in circulating myokines after completion of a 12-week exercise training program among postmenopausal women with obesity and at heightened risk of breast cancer.

Acknowledgements We would like to thank to the NCI R25 Cancer Prevention Research Training Program for support of AMC (R25TCA057730, PI: Shine Chang PhD), the T32 Cancer Biology Training Grant for support of GMN (T32CA009213, PI: Anne Cress, $\mathrm{PhD}$ ), the University of Texas MD Anderson Cancer Center/Energy Balance Assessment Supplemental Funding (PI: Susan Gilchrist MD), the MD Anderson Cancer Center, Center for Energy Balance in Cancer Prevention and Survivorship, and the MD Anderson Cancer Center Duncan Family Institute for Cancer Prevention and Risk Assessment.

Author contributions Conceptualization: SCG, RJS; data curation: AMC; formal analysis: AMC; funding acquisition: SCG, RJS, AMC; investigation: SCG, RJS, AMC, NHA, FLB, GMN, PLM, EF-M; methodology: SCG, RJS, EF-M; project administration: SCG, AMC; resources: SCG, RJS, AMC, AMB, TBB, EF-M, KB-E; supervision: SCG; writing — original draft: AMC; writing—review \& editing: all authors. All authors read and approved the final manuscript.

Funding We would like to thank to the NCI R25 Cancer Prevention Research Training Program for support of AMC (R25TCA057730, PI: Shine Chang PhD), the T32 Cancer Biology Training Grant for support of GMN (T32CA009213, PI: Anne Cress, PhD), the University of Texas MD Anderson Cancer Center/Energy Balance Assessment Supplemental Funding (PI: Susan Gilchrist MD), the MD Anderson Cancer Center, Center for Energy Balance in Cancer Prevention and Survivorship, and the MD Anderson Cancer Center Duncan Family Institute for Cancer Prevention and Risk Assessment.

Data availability All data generated or analyzed during this study are available upon request to the corresponding author.

\section{Compliance with ethical standards}

Conflict of interest The authors declare that they have no conflict of interest.

Ethical Approval All procedures performed in studies involving human participants were in accordance with the ethical standards of the University of Texas MD Anderson Cancer Center Institutional Review Board (IRB: 2016-0442) and with the 1964 Helsinki declaration and its later amendments or comparable ethical standards.

Informed consent Informed consent was obtained from all individual participants included in the study.

Open Access This article is licensed under a Creative Commons Attribution 4.0 International License, which permits use, sharing, adaptation, distribution and reproduction in any medium or format, as long as you give appropriate credit to the original author(s) and the source, provide a link to the Creative Commons licence, and indicate if changes were made. The images or other third party material in this article are included in the article's Creative Commons licence, unless indicated otherwise in a credit line to the material. If material is not included in the article's Creative Commons licence and your intended use is not permitted by statutory regulation or exceeds the permitted use, you will need to obtain permission directly from the copyright holder. To view a copy of this licence, visit http://creativecommons.org/licenses/by/4.0/. 


\section{References}

1. Weston KS, Wisloff U, Coombes JS (2014) High-intensity interval training in patients with lifestyle-induced cardiometabolic disease: a systematic review and meta-analysis. Br J Sports Med 48:1227-1234

2. Coletta AM, Brewster AM, Chen M et al (2019) High-intensity interval training is feasible in women at high risk for breast cancer. Med Sci Sports Exerc 51(11):2193-2200

3. Devin J, Jenkins D, Sax A et al (2018) Cardiorespiratory fitness and body composition responses to different intensities and frequencies of exercise training in colorectal cancer survivors. Clin Colorectal Cancer. https://doi.org/10.1016/j.clcc.2018.01.004

4. Devin J, Sax A, Hughes G et al (2017) The influence of highintensity compared with moderate-intensity exercise training on cardiorespiratory fitness and body composition in colorectal cancer survivors: a randomised controlled trial. J Cancer Surviv $10: 467-479$

5. Godin GDR, Valois P, Lepage P, Jobin J, Bradet R (1994) Differences in perceived barriers to exercise between high and low intenders: observations among different populations. Am J Health Promot 8:279-285

6. Sprague BLT-DA, Newcomb PA, Titus-Ernstoff L, Hampton JM, Egan KM (2007) Lifetime recreational and occupational physical activity and risk of in situ and invasive breast cancer. Cancer Epidemiol Biomark Prev 16(2):236-243

7. Bray F, Ferlay J, Soerjomataram I, Siegel RL, Torre LA, Jemal A (2018) Global cancer statistics 2018: GLOBOCAN estimates of incidence and mortality worldwide for 36 cancers in 185 countries. CA Cancer J Clin. https://doi.org/10.3322/caac.21492

8. Tamimi RM, Spiegelman D, Smith-Warner SA et al (2016) Population attributable risk of modifiable and nonmodifiable breast cancer risk factors in postmenopausal breast cancer. Am J Epidemiol 184(12):884-893

9. Dyrstad SW, Yan Y, Fowler AM, Colditz GA (2015) Breast cancer risk associated with benign breast disease: systematic review and meta-analysis. Breast Cancer Res Treat 149:569-575

10. Lauby-Secretan B, Scoccianti C, Loomis D et al (2016) Body fatness and cancer-viewpoint of the IARC Working Group. N Engl J Med 375(8):794-798

11. Hojman P, Dethlefsen C, Brandt C, Hansen J, Pedersen L, Pedersen BK (2011) Exercise-induced muscle-derived cytokines inhibit mammary cancer cell growth. Am J Physiol Endocrinol Metab 301(3):E504-510

12. Hojman P, Gehl J, Christensen JF, Pedersen BK (2018) Molecular mechanisms linking exercise to cancer prevention and treatment. Cell Metab 27(1):10-21

13. Duggal NA, Niemiro G, Harridge SDR, Simpson RJ, Lord JM (2019) Can physical activity ameliorate immunosenescence and thereby reduce age-related multi-morbidity? Nat Rev Immunol. https://doi.org/10.1038/s41577-019-0177-9

14. Duggal NA, Niemiro G, Harridge SDR, Simpson RJ, Lord JM (2019) Can physical activity ameliorate immunosenescence and thereby reduce age-related multi-morbidity? Nat Rev Immunol 19(9):563-572

15. Gannon NP, Vaughan RA, Garcia-Smith R, Bisoffi M, Trujillo KA (2015) Effects of the exercise-inducible myokine irisin on malignant and non-malignant breast epithelial cell behavior in vitro. Int J Cancer 136(4):E197-202

16. Fox J, Rioux BV, Goulet EDB et al (2018) Effect of an acute exercise bout on immediate post-exercise irisin concentration in adults: a meta-analysis. Scand J Med Sci Sports 28(1):16-28

17. Provatopoulou X, Georgiou GP, Kalogera E et al (2015) Serum irisin levels are lower in patients with breast cancer: association with disease diagnosis and tumor characteristics. BMC Cancer. https://doi.org/10.1186/s12885-015-1898-1

18. Idorn M, Hojman P (2016) Exercise-dependent regulation of NK cells in cancer protection. Trends Mol Med 22(7):565-577

19. Pedersen L, Idorn M, Olofsson GH et al (2016) Voluntary running suppresses tumor growth through epinephrine- and IL6 -dependent NK cell mobilization and redistribution. Cell Metab 23(3):554-562

20. Bigley AB, Rezvani K, Chew $C$ et al (2014) Acute exercise preferentially redeploys NK-cells with a highly-differentiated phenotype and augments cytotoxicity against lymphoma and multiple myeloma target cells. Brain Behav Immun 39:160-171

21. Dethlefsen C, Pedersen KS, Hojman P (2017) Every exercise bout matters: linking systemic exercise responses to breast cancer control. Breast Cancer Res Treat 162(3):399-408

22. Kresovich JK, O'Brien KM, Xu Z, Weinberg CR, Sandler DP, Taylor JA (2020) Prediagnostic immune cell profiles and breast cancer. JAMA Netw open 3(1):e1919536

23. Pescatello LS, American College of Sports M (2014) ACSM's guidelines for exercise testing and prescription. Wolters Kluwer/ Lippincott Williams \& Wilkins Health, Philadelphia

24. Maeng HG, Lee SJ, Lee YA et al (2018) Hemacytotoxicity and natural killer lytic index: new parameters to evaluate natural killer cell immunity for clinical use in cancer. Oncol Lett 15(1):1325-1333

25. Bigley AB, Agha NH, Baker FL et al (2018) NK-cell function is impaired during long-duration spaceflight. J Appl Physiol 126:842-853

26. Yoon JR, Ha GC, Ko KJ, Kang SJ (2018) Effects of exercise type on estrogen, tumor markers, immune function, antioxidant function, and physical fitness in postmenopausal obese women. J Exerc Rehabil 14(6):1032-1040

27. Campbell PT, Wener MH, Sorensen B et al (2008) Effect of exercise on in vitro immune function: a 12-month randomized, controlled trial among postmenopausal women. J Appl Physiol 104(6):1648-1655

28. Pal A, Zimmer P, Schmidt ME et al (2019) No evidence for effect of exercise on transcriptome of NK cells in breast cancer patients undergoing adjuvant therapy: results from a pilot study. Front Physiol. https://doi.org/10.3389/fphys.2019.00959

29. Moro-García MA, Fernández-García B, Echeverría A et al (2014) Frequent participation in high volume exercise throughout life is associated with a more differentiated adaptive immune response. Brain Behav Immun 39:61-74

30. Nieman DC, Buckley KS, Henson DA et al (1995) Immune function in marathon runners versus sedentary controls. Med Sci Sports Exerc 27(7):986-992

31. Pedersen BK, Tvede N, Christensen LD, Klarlund K, Kragbak S, Halkjr-Kristensen J (1989) Natural killer cell activity in peripheral blood of highly trained and untrained persons. Int J Sports Med 10(2):129-131

32. Aoi W, Naito Y, Takagi T et al (2013) A novel myokine, secreted protein acidic and rich in cysteine (SPARC), suppresses colon tumorigenesis via regular exercise. Gut 62(6):882-889

33. Comes A, Di Carlo E, Musiani P et al (2002) IFN- $\gamma$-independent synergistic effects of IL-12 and IL-15 induce anti-tumor immune responses in syngeneic mice. Eur J Immunol 32:1914-1923

34. Pedersen BK (2011) Exercise-induced myokines and their role in chronic diseases. Brain Behav Immun 25(5):811-816

35. Matsuo K, Sato K, Suemoto K et al (2017) A mechanism underlying preventive effect of high-intensity training on colon cancer. Med Sci Sports Exerc 49(9):1805-1816

36. Eaton M, Granata C, Barry J, Safdar A, Bishop D, Little JP (2018) Impact of a single bout of high-intensity interval exercise and short-term interval training on interleukin-6, FNDC5, and 
METRNL mRNA expression in human skeletal muscle. J Sport Health Sci 7(2):191-196

37. Huh JY, Panagiotou G, Mougios V et al (2012) FNDC5 and irisin in humans: I. Predictors of circulating concentrations in serum and plasma and II. mRNA expression and circulating concentrations in response to weight loss and exercise. Metabolism 61(12):1725-1738
38. Qiu S, Cai X, Sun Z, Schumann U, Zügel M, Steinacker JM (2015) Chronic exercise training and circulating Irisin in adults: a metaanalysis. Sports Med 45(11):1577-1588

Publisher's Note Springer Nature remains neutral with regard to jurisdictional claims in published maps and institutional affiliations.

\section{Authors and Affiliations}

\section{Adriana M. Coletta ${ }^{1,2,12}$ - Nadia H. Agha ${ }^{3}$. Forrest L. Baker ${ }^{4,5}$. Grace M. Niemiro ${ }^{4}$. Preteesh L. Mylabathula ${ }^{4,5}$. Abenaa M. Brewster ${ }^{6} \cdot$ Therese B. Bevers $^{6}$. Enrique Fuentes-Mattei ${ }^{7} \cdot$ Karen Basen-Engquist $^{8}$. Susan C. Gilchrist ${ }^{6,9}$. Richard J. Simpson ${ }^{4,5,8,10,11}$}

1 Department of Health and Kinesiology, The University of Utah, Salt Lake City, UT, USA

2 Cancer Control and Population Sciences Program, Huntsman Cancer Institute, Salt Lake City, UT, USA

3 Department of Health and Human Performance, The University of Houston, Houston, TX, USA

4 Department of Pediatrics, The University of Arizona, Tucson, AZ, USA

5 Department of Nutritional Sciences, The University of Arizona, Tucson, AZ, USA

6 Department of Clinical Cancer Prevention, The University of Texas MD Anderson Cancer Center, Houston, TX, USA
7 Department of Radiation Oncology Clinical Research, The University of Texas MD Anderson Cancer Center, Houston, TX, USA

8 Department of Behavioral Science, The University of Texas MD Anderson Cancer Center, Houston, TX, USA

9 Department of Cardiology, The University of Texas MD Anderson Cancer Center, Houston, TX, USA

10 Department of Immunobiology, The University of Arizona, Tucson, AZ, USA

11 The University of Arizona Cancer Center, Tucson, AZ, USA

12 Huntsman Cancer Institute at the University of Utah, 2000 Circle of Hope Drive, Research South Building Room 4747, Salt Lake City, UT 84112, USA 